\title{
On the completeness of sets of complex exponentials
}

\author{
Kevin Smith
}

\begin{abstract}
The completeness of sets of complex exponentials $\left\{e^{i \lambda f(n) \cdot}: f \in \mathbb{Z}\right\}$ in $L^{p}(-\pi, \pi), 1<p \leqslant 2$, is considered under Levinson's sufficient condition in the non-trivial case $\lambda \geqslant 1-(1 / p)$. All such sets are determined explicitly.
\end{abstract}

\section{Introduction}

In 1934, Paley and Wiener [2] proved that a set of complex exponentials $\left\{e^{i \lambda_{n} \cdot}\right\},\left\{\lambda_{n}\right\} \subset \mathbb{R}$, is complete on $L^{2}(-\pi, \pi)$ if there exists a constant $D<\pi^{-2}$ such that

$$
\left|\lambda_{n}-n\right| \leqslant D
$$

for every $n \in \mathbb{Z}$. Shortly after, Levinson $[1]$ considered the $L^{p}(-\pi, \pi)$ case with $1<p \leqslant 2$ and $1 / p+1 / q=1$. He proved that completeness holds on $L^{p}(-\pi, \pi)$ if

$$
\left|\lambda_{n}-n\right| \leqslant D<1 / 2 q,
$$

and that this result is optimal in the sense that completeness may fail if $D=1 / 2 q$.

In this short paper we consider the existence of solutions to Levinson's inequality (1.2) which are subsequences of the scaled integers $\lambda \mathbb{Z}, \lambda \in \mathbb{R}$, in other words, we consider commensurable solutions. In this case, the problem reduces to finding $f: \mathbb{Z} \rightarrow \mathbb{Z}$ such that $\lambda_{n}=\lambda f(n)$. The structure of the solutions and the action of $f$ on the additive group $\mathbb{Z} /(2 r+1) \mathbb{Z}$ are discussed once the reader has been introduced to the relevant concepts via a proof of the main theorem.

For $0<\lambda<1 / q$, the existence of $f$ is trivial because for every $n \in \mathbb{Z}$ there exists at least one $m \in \mathbb{Z}$ such that $|\lambda m-n|<\lambda / 2$. On the other hand, for $\lambda \geqslant 1 / q, f$ is injective if it exists.

\section{Main theorem}

The special case $p=q=2$ was solved in $[3]$ via a complicated and laborious algorithm. We shall now prove the general theorem for $1<p \leqslant 2$ using elementary principles from group theory, which demonstrate more clearly why the structure of the solutions is as such. Specifically (a non-trivial case being one in which $\lambda f(n) \neq n$ for at least one $n$ ), we shall prove the following theorem.

TheOREm 2.1. If $\lambda \geqslant 1 / q$, there exists a non-trivial $f: \mathbb{Z} \rightarrow \mathbb{Z}$ such that

$$
|\lambda f(n)-n| \leqslant D<1 / 2 q
$$

if and only if $\lambda=(2 r+1) / s$ is an irreducible fraction with $s=2 q r+t$ and $1 \leqslant t<q$.

Proof. We begin by excluding the possibility that $\lambda$ is irrational or rational with an even numerator. By Kronecker's approximation theorem, the fractional parts of the set $\lambda \mathbb{Z}$ are dense in the unit interval $(0,1)$. It follows that an irrational $\lambda$ cannot solve $(1.2)$, so $\lambda=r / s$, where $r, s \in \mathbb{N}$, and, since $f$ is one-to-one, we must have $f(r)=s$, so $\lambda=r / f(r)$. To exclude the possibility that the numerator is even, suppose that $\lambda=2 r / s$ is irreducible, 
so that $f(2 r) \neq 2 f(r)$. Dividing (2.1) by $\lambda n=2 r n / f(2 r)$, we obtain the weaker condition

$$
\left|\frac{f(n)}{n}-\frac{f(2 r)}{2 r}\right| \leqslant C<\frac{1}{2 n} \text {. }
$$

Now, since $f(2 r) \neq 2 f(r)$ and $f: \mathbb{Z} \rightarrow \mathbb{Z}$, we have $|f(2 r)-2 f(r)| \geqslant 1$, so $|f(2 r) / 2 r-f(r) / r| \geqslant$ $1 / 2 r$, a contradiction. We must conclude that the numerator is odd, so $\lambda=(2 r+1) / s, r, s \in \mathbb{N}$.

The fact that $\lambda$ must be rational with an odd numerator means that we may replace $|\cdot| \leqslant D<1 / 2 q$ by $|\cdot|<1 / 2 q$, and work modulo $2 r+1$ because $f$ is actually a function on the additive group $\mathbb{Z} /(2 r+1) \mathbb{Z}$, that is, $f(n)=f(j(2 r+1)+k)=j f(2 r+1)+f(k)=$ $f(k) \bmod f(2 r+1)$. Dividing $(2.1)$ by $\lambda \geqslant 1 / q$, we obtain the weaker condition

$$
\left|f(n)-\frac{n}{\lambda}\right|<\frac{1}{2}
$$

so that $f(n)=\lfloor n / \lambda+1 / 2\rfloor$ is the nearest integer to $n / \lambda$ in any case.

Let $s=m(2 r+1)+l$. Since we need only consider $\lambda$ irreducible, we have $(2 r+1, s)=$ $(2 r+1, l)=1$ and

$$
\begin{aligned}
g(k s) & =k s-(2 r+1)\left\lfloor\frac{k s}{2 r+1}+\frac{1}{2}\right\rfloor \\
& =k l-(2 r+1)\left\lfloor\frac{k l}{2 r+1}+\frac{1}{2}\right\rfloor \\
& =k l \quad \bmod 2 r+1 .
\end{aligned}
$$

For each $k \in[-r, r]$, the map $k \rightarrow k l, l \in[-r, r]$, is incongruent $\bmod 2 r+1$, and, since $g$ is the corresponding element in the least residue system $[-r, r], g$ defines $\phi(2 r+1)+1$ automorphisms of the additive group $\mathbb{Z} /(2 r+1) \mathbb{Z}$. Since $\lambda \geqslant 1 / q$ is irreducible, we have $(2 r+1, s)=1$ and it follows that $g$ takes the values $\pm r$ independently of $s$, so

$$
\sup _{n \in \mathbb{Z}}|\lambda f(n)-n|=\sup _{n \in \mathbb{Z}}\left|\frac{g(n s)}{s}\right|=\frac{r}{s}
$$

and (2.1) holds if and only if $s=2 q r+t, 1 \leqslant t<q$, which completes the proof.

\section{Remarks on the action of $f$ on the additive group $\mathbb{Z} /(2 r+1) \mathbb{Z}$}

In the course of proving Theorem 2.1 we found that, for each solution $\lambda$, we must have $f(k)=$ $\lfloor k / \lambda+1 / 2\rfloor$. The assertion of the theorem may be expressed as $s=q(2 r+1)+l, 1-q \leqslant l<0$, which, on substitution into the expression for $f$, gives $f(k)=k q+\lfloor k l /(2 r+1)+1 / 2\rfloor$. Since $k \in[-r, r]$, this implies that $f: k \rightarrow k q$ if and only if $l=-1$, that is, $\lfloor k l /(2 r+1)+1 / 2\rfloor=0$, which is exactly the case in which $\lambda$ is the best possible rational approximation to $1 / q$ with numerator $2 r+1$. It is interesting to note that this is the only case in which $f$ is an automorphism of the additive group $\mathbb{Z} /(2 r+1) \mathbb{Z}$.

For finite $q$, the solutions corresponding to the remaining congruences $l=-2, \ldots, 1-q$ do not yield functions which preserve the structure of the group. Yet, it is interesting to note that the only possibility in the $L^{1}$ (that is, $q=\infty$ ) and $L^{2}$ cases are the functions $f: k \rightarrow k$ and $f: k \rightarrow 2 k$, respectively, which are both automorphisms of $\mathbb{Z} /(2 r+1) \mathbb{Z}$.

It also seems worth remarking that for sufficiently large $r$, specifically $2 r+1>q$, we find that there are exactly $q-1$ distinct non-trivial solutions, and hence $q-1$ distinct non-trivial functions $f$. Therefore, including the trivial (identity) case $f(n)=n$, one has $q=(1-(1 / p))^{-1}$ distinct solutions. This fact easily follows from the observation that, for every $1 \leqslant t<q, 2 r q+t$ is not divisible by $2 r+1$ if $2 r+1>q$.

Acknowledgements. The author wishes to thank A. Granville, J. R. Higgins, J. Morrison, T. Wesson and an anonymous referee for valuable discussions and suggestions. 


\section{References}

1. N. Levinson, 'On non-harmonic Fourier series', Ann. of Math. (2) 37 (1936) 919-936.

2. R. E. A. C. PAley and N. Wiener, Fourier transforms in the complex domain (American Mathematical Society, Providence, RI, 1934).

3. K. Sмiтн, 'On complete interpolating sequences and sampling expansions', LMS J. Comput. Math. 13 (2010) 1-9.

Kevin Smith

Cambridge

United Kingdom

k.p.q.smith@gmail.com 